Imaging of molybdenum erosion and thermography at visible wavelengths in Alcator C-Mod ICRH and LHCD discharges

A. N. James, D. Brunner, B. LaBombard, C. Lau, B. Lipschultz, D. Miller, M. L. Reinke, J. L. Terry, C. Theiler, G. M. Wallace, D. G. Whyte, S. Wukitch, V. Soukhanovskii

June 6, 2013 
This document was prepared as an account of work sponsored by an agency of the United States government. Neither the United States government nor Lawrence Livermore National Security, LLC, nor any of their employees makes any warranty, expressed or implied, or assumes any legal liability or responsibility for the accuracy, completeness, or usefulness of any information, apparatus, product, or process disclosed, or represents that its use would not infringe privately owned rights. Reference herein to any specific commercial product, process, or service by trade name, trademark, manufacturer, or otherwise does not necessarily constitute or imply its endorsement, recommendation, or favoring by the United States government or Lawrence Livermore National Security, LLC. The views and opinions of authors expressed herein do not necessarily state or reflect those of the United States government or Lawrence Livermore National Security, LLC, and shall not be used for advertising or product endorsement purposes.

This work performed under the auspices of the U.S. Department of Energy by Lawrence Livermore National Laboratory under Contract DE-AC52-07NA27344. 
LLNL IM release: LLNL-TR-638555

PSFC Report: PSFC/JA-13-24

\title{
Imaging of molybdenum erosion and thermography at visible wavelengths in Alcator C-Mod ICRH and LHCD discharges
}

\author{
A. N. James, ${ }^{1, a)}$ D. Brunner, ${ }^{2}$ B. Labombard, ${ }^{2}$ C. Lau, ${ }^{2}$ B. Lipschultz, ${ }^{2}$ D. Miller, ${ }^{2}$ M. \\ L. Reinke, ${ }^{2}$ J. L. Terry, ${ }^{2}$ C. Theiler, $^{2}$ G. M. Wallace, ${ }^{2}$ D. G. Whyte, ${ }^{2}$ S. Wukitch, ${ }^{2}$ and \\ V. Soukhanovskii ${ }^{1}$ \\ 1) Lawrence Livermore National Laboratory, Fusion Energy Sciences Program \\ ${ }^{2)}$ Massachusetts Institute of Technology, Plasma Science and Fusion Center
}

(Dated: 20 June 2013)

We describe results from imaging observations of atomic line and continuum emission in the 550.6nm region on Alcator C-Mod. Both the 550.6nm neutral molybdenum emission and the adjacent $549 \mathrm{~nm}$ continuum emission are imaged separately to isolate line emission. A few complications of using imaging to infer erosion in this wavelength region are discussed including subtraction of continuum emission and determination of an appropriate $\mathrm{S} / \mathrm{XB}$ coefficient. Diagnostics of surface erosion and thermography using these emissions are briefly reviewed, and used to study phenomenology during ohmic operation, ion cyclotron range of frequencies heating (ICRH), and lower hybrid current drive (LHCD). In addition to broadening of Mo I emission regions in the outer divertor and main limiter during ICRH compared to ohmic operation, mid-plane localized heating of the main limiter associated with fast ion impact is observed which exceeds the divertor heat flux. During LHCD operation, several localized regions of increased brightness associated with hot-spots are interpreted as heating due to localized density peaking, which re-iterates the importance of imaging continuum emission for subtraction. These sources of surface heating exacerbate plasma-material interactions at the device wall and may require additional mitigation if they cannot be avoided in future machines.

\footnotetext{
a)Electronic mail: atronchi@psfc.mit.edu; http://psfc.mit.edu/ atronchi/
} 


\section{INTRODUCTION}

The spatially complex plasma-material interactions (PMI) in magnetic fusion devices which can deposit excess heat and particle fluxes causing respective melting and erosion of plasma facing components (PFC's) requires the use of imaging diagnostics to understand experimental operations. In future magnetic fusion devices, imaging diagnostics must overcome difficulties which do not occur in many present machines.

Imaging diagnostics are increasingly deployed at present ${ }^{1,2}$ and future ${ }^{3,4}$ devices to observe erosion, heating, and other key PMI phenomena ${ }^{5-8}$ which are often toroidally asymmetric. In addition, imaging diagnostics will likely be used in future machines for realtime monitoring and feedback control of PFC conditions ${ }^{9}$. In many modern tokamak experiments, visible continuum emission is negligible compared to line emission over most of the experimental view, so that subtraction is not necessary to infer the erosion source. High power and high density experiments will have more substantial continuum sources however, pressing the need to develop diagnostic techniques to separate continuum and line emission in imaging diagnostics.

High Z (atomic number) refractory metals such as tungsten are under investigation as potential PFC materials in fusion reactors due to their low erosion yields, high melting points, and resistance to activation and damage from neutron fluence ${ }^{10-14}$. Molybdenum has similar properties to tungsten for studies in a non-nuclear environment, but is an undesirable material for reactors due to propensity for transmutation. Spectroscopic and imaging techniques have been used to diagnose erosion of these materials, but deficiencies in atomic modeling ${ }^{15}$ limit their experimental application.

In steady state devices, actuators such as ion-cyclotron range of frequencies heating (ICRH) and lower hybrid current drive (LHCD) are two potential heating and current drive mechanisms ${ }^{16}$ as opposed to ohmic heating and current drive. During certain operating conditions, these actuators have been observed to cause additional PMI ${ }^{9,17,18}$. Imaging diagnostics have also been beneficial for investigating these phenomena.

The Alcator C-Mod tokamak is the only United States experiment presently addressing all of these topics. C-Mod is a compact device with high values of magnetic field, density, temperature, and power/particle flux, all similar to projected reactor relevant quantities ${ }^{19-21}$. The plasma-material interface at C-Mod is composed entirely of molybdenum, with the first 
ever vertical plate lower divertor, a toroidally continuous inner wall limiter, an outer wall main limiter of limited toroidal extent, and several smaller limiter surfaces typically of limited toroidal and poloidal extent for protecting antenna structures and other in-vessel hardware. At C-Mod, an intensified charge injection device (CID) camera is installed and used to monitor erosion and surface temperature of PFC surfaces during operation of ICRH and LHCD. Design of this system and interpretation of observations are described in detail in this article.

Section II describes the experiment layout. Interpretation of spectroscopic data is reviewed in section III. Initial results from the new camera diagnostic are presented and discussed in section IV. Lastly, some implications of the observations and potential future improvements to the diagnostic techniques are briefly discussed in section V. Two appendices describe the camera system in detail and a quasi-emipirical determination of an $\mathrm{S} / \mathrm{XB}$ value respectively.

\section{EXPERIMENT LAYOUT}

This article describes first results from a charge injection device (CID) camera mounted with an intensifier and narrow band interference filters, used to image continuum and atomic line emission as discussed in section III. The camera is mounted roughly $2.5 \mathrm{~m}$ from the tokamak axis and $1 \mathrm{~m}$ above the mid-plane to minimize exposure to hard x-ray radiation and magnetic field. Radiation noise on the camera is generally undetectable in this configuration at C-Mod, however the image is noticeably perturbed when vertical magnetic fields at the camera exceed roughly $0.05 \mathrm{~T}$. Figure 1 shows the camera view which spans essentially four distinct regions: the inner wall, the main $(\mathrm{GH})$ limiter, and the outer part of the lower divertor, with a heating antenna protection tile in the foreground. The camera system is described in detail in appendix A.

A raw camera frame viewed through a narrow-pass interference filter centered at $550.6 \mathrm{~nm}$ during plasma startup in un-boronized conditions is also shown in figure 1. Since the plasma is inner-wall limited during startup, strong emission is observed at the machine mid-plane. Though most of the tiles on the main limiter are installed with thick boron coatings, some emission is still observed there, probably due to molybdenum being re-deposited to the limiter from other regions. 


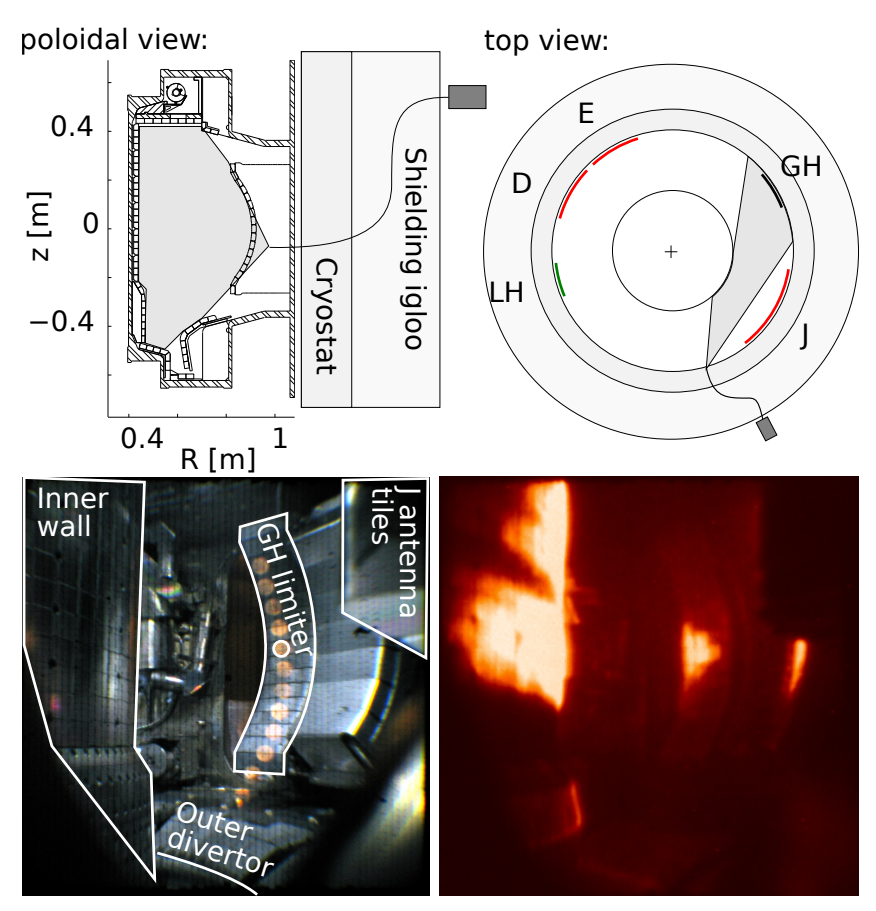

FIG. 1. Above: A schematic of the camera view at Alcator C-Mod. ICRH antennas are shown in red, and the lower hybrid (LH) launcher is shown in green. Below: Images through the camera view with left: annotations and backlit spectrometer views, and right: an image recorded during plasma startup.

Ultraviolet to visible range spectroscopy is achieved using a Chromex 250IS spectrograph with a $600 / \mathrm{mm}$ grooved grating producing spectral dispersion of roughly $0.07 \mathrm{~nm}$ per pixel. This spectrometer has views of the main limiter from the same periscope as the camera along roughly the same line of sight (shown backlit in figure 1), and also of the divertor region.

The camera and spectrometer systems were absolutely calibrated by transfer from a LPS-200 Labsphere with a URS-600 source. At the typical intensifier gain (50\%) and gate $(18.8 \mathrm{~ms})$ settings used to obtain data, the camera is sensitive to band integrated radiances of $0.051-16.0 \mu W / \mathrm{cm}^{2} \mathrm{sr}$. Thin boron deposits on optical surfaces in C-Mod can alter this calibration, so the data quantitatively discussed below were all selected near to the calibration date to minimize boron accumulation between the experiment and post-calibration.

An array of fixed Langmuir probes ${ }^{22}$ are used to measure the electron temperature and density at plasma facing surfaces. Densely packed probe arrays enable measurement of the 
spatially peaked temperature and density at the divertor strike points. Though the entire inner wall is not spanned by a probe array, the highest two probes of the lower inner divertor array (at the bottom of the inner wall) generally measure comparable signals. This indicates a weak gradient along the inner wall, so these measurements are used to represent the entire inner wall, assuming no gradients. While the main limiter is not directly instrumented with probes, a set of probes are mounted on the lower hybrid launcher near the mid-plane and recessed approximately $2 \mathrm{~mm}$ behind the limiter. Interaction between these outer mid-plane probes and strong RF potentials during ICRH causes strong fluctuation of the currentvoltage characteristic curves, making them useless for measurements during this time.

The Langmuir probes and camera and spectrometer views are generally toroidally spaced from the observed surfaces, requiring that we assume toroidal symmetry of the measured quantities. This assumption can be violated by the presence of homoclinic tangles ${ }^{23}$, however these phenomena are not apparent in divertor region imaging for these experiments. RF actuators can also cause toroidally asymmetric perturbations, so where any field aligned signatures are observed we must discard this assumption.

These diagnostics are used in combination to observe the plasma-wall interactions in C-Mod.

\section{DATA INTERPRETATION}

Light observed by the camera comes from a combination of mechanisms, including atomic line emission and multiple sources of continuum emission: Planck emission, visible bremsstrahlung, and molecular continuum. By separating the continuum sources from line emission and considering the conditions in which various continuum sources will dominate observed light, the surface erosion rate and temperature can be diagnosed as described below.

\section{A. Atomic line emission}

Through various edge transport processes, hot plasma interacts with the first wall PFC's and sputters neutral atoms from the surface. These atoms are then excited primarily by thermal electron impact from the same hot plasma, causing photon emission. In the follow- 
ing, we discuss how measurements of this emission are used for diagnosis of plasma-surface interactions.

\section{Molydenum line selection}

Though the camera system itself is optimized for UV sensitivity, strong absorption below about $400 \mathrm{~nm}$ in the fiber bundle after modest radiation damage ${ }^{24}$ limits the lower wavelength observable by the system, and low sensitivity of the intensifier photocathode above $800 \mathrm{~nm}$ establishes an upper bound. Neutral molybdenum has several lines in the visible wavelength range, with line triplets commonly used for erosion studies near $390 \mathrm{~nm}, 410 \mathrm{~nm}$, and $550 \mathrm{~nm}$. While the 390nm lines are brightest, the lowest molybdenum reflectivity occurs there ${ }^{25}$, and atomic modeling of the associated ground state transitions is accurate ${ }^{15}$, the low fiber bundle transmission at that range limits the value of these lines for erosion imaging with the present setup. The nearby 410nm lines are much lower intensity, and so also of limited value.

At $550 \mathrm{~nm}$, the line intensity is still moderately strong and the fiber bundle transmission is as high as $35 \%$ before irradiation, so this triplet was determined most suitable given the available equipment. Drawbacks of this selection include a greater reflectivity at visible compared to UV wavelengths and stronger Planck emission for fixed temperature at longer wavelengths. Atomic modeling for the metastable transitions associated with these lines is more difficult and less accurate than modeling for better understood ground state transitions. These are all issues that must be acknowledged for future devices, where the nuclear environment will impose hard limits which data interpretation must accommodate, despite the added complexity.

Interfering lines occur in this region due to at least nitrogen gas puffing (N II, 549.566nm), which is used in some divertor studies ${ }^{26}$. Neither neon gas puffing at levels relevant for erosion suppression experiments, nor argon gas puffing at levels needed to measure ion temperature and rotation profiles ${ }^{27}$ appear to produce much interfering line emission. During tungsten injections believed to result from the plasma edge interacting with Langmuir probes, only minor neutral tungsten emission (W I, 551.468nm) was observed except during accidental 'killer' injections. A lithium line (Li II, 549.5nm) also appears nearby which is only of concern where lithium is used as an alternative to boron for wall conditioning (i.e. NSTX).

The 550nm molybdenum triplet and known interfering lines are shown in figure 2 for ref- 
erence. Based on these observations an interference filter with center wavelength at 550.6nm was selected to image the desired Mo I line. A filter width of $1 \mathrm{~nm}$ was selected to maximize line emission throughput vs. continuum emission, and to reject spectrally nearby lines.

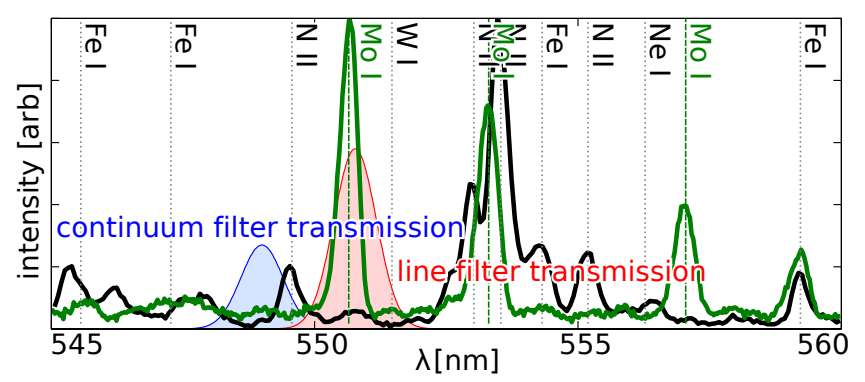

FIG. 2. Spectral range data showing filter transmissions, molybdenum lines, interfering nitrogen lines from the chromex visible range spectrometer at C-Mod. Line intensities are normalized to the maximum for each spectrum.

\section{Inferring erosion fluxes}

Particle influx from the surface $\Gamma$ can be inferred using the following expression ${ }^{28}$ :

$$
\Gamma=4 \pi I(S / X B)
$$

The absolutely calibrated camera system measures line intensity $I$ in units of photons $/ \mathrm{cm}^{2} \mathrm{sr} / \mathrm{s}$. $\mathrm{S} / \mathrm{XB}$ coefficients must be determined from either experiments or atomic modeling, and the electron temperature and density at PFC surfaces across the field of view must also be imaged somehow. Since discrepancies exist between atomic modeling and experimental determination of some S/XB coefficients, an improved coefficient can be empirically determined as described in appendix B. In these experiments, no imaging temperature or density measurement existed to calculate the $\mathrm{S} / \mathrm{XB}$ coefficient, so the quantity $4 \pi I=\Gamma_{M o} /(S / X B)$ is shown where appropriate.

\section{B. Continuum emission}

In the high density and high power conditions at Alcator C-Mod, continuum emission can dominate atomic line emission along certain views. Visible bremsstrahlung (VB) from core 
impurities, molecular emission in the cold boundary, and Planck emission from hot plasma facing components have all been observed, as shown in figure 3. These sources can exceed line emission in some regions and circumstances, so continuum emission must be subtracted from the line emission image. Since continuum sources can vary rapidly in both space and in time, this subtraction is not as simple as measuring the resolved spectrum at a few points to fit the continuum and line emission contributions, but requires separate imaging.

To interpret observed light as an eroded particle flux, these continuum sources must be separated from atomic line emission. This is accomplished here by imaging a line-free region spectrally adjacent to the atomic line of interest but close enough that the intensity can be assumed similar. An interference filter with bandpass wavelength centered at 549nm was selected for this purpose, with the same $1 \mathrm{~nm}$ width to match the line emission filter, as shown in figure 3. The two filters were switched between two sequential similar discharges to record an image through both the line filter and continuum filter along the same optical view, so that the two images could be subtracted to isolate Mo I emission. The various known sources of continuum emission and their application for plasma diagnostics are discussed in further detail below.

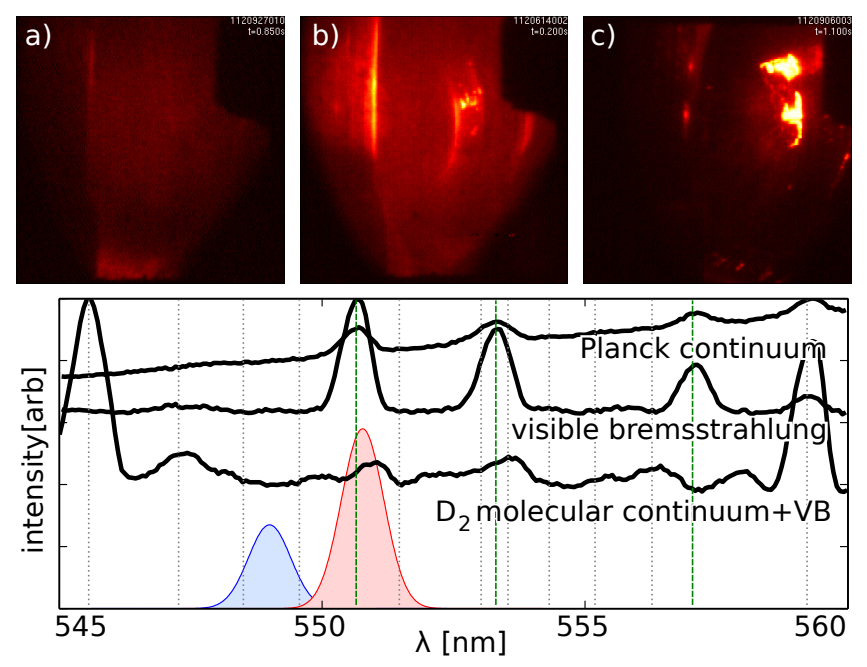

FIG. 3. Camera images through the $549 \mathrm{~nm}$ continuum filter during periods when emission is mostly dominated by a) VB, b) molecular emission, and c) Planck emission. Below are spectral range data showing filter transmissions, molybdenum lines, and typical continuum observations. Line intensities are normalized to maximum for each spectrum. 


\section{Planck emission and inferring temperature}

Thermography via radiated power is a well established technique ${ }^{29,30}$, with the in-band spectral radiance at center wavelength $\lambda$, bandwidth $\Delta \lambda$ and temperature $T$ described by Planck's law with isotropic black-body emissivity assumed:

$$
B_{P l}(\lambda)=\frac{1}{2 \pi} \frac{2 h c^{2}}{\lambda^{5}} \frac{1}{\exp \left(\frac{h c / \lambda}{k T}\right)-1} \Delta \lambda
$$

Though clean molybdenum is known to have a low emissivity $(\sim 0.2)^{31}$, boron and other coatings which accumulate during operation tend to raise the emissivity, such that our inferred temperatures should be taken as a lower bound. For typical camera settings, a negligible contribution from Planck emission occurs for temperatures below $1330 \mathrm{~K}$, corresponding to in-band radiance of about $0.1 \mu \mathrm{W} / \mathrm{cm}^{2} \mathrm{sr}$. Above about $1850 \mathrm{~K}$, the in-band Planck emission radiance of $26 \mu \mathrm{W} / \mathrm{cm}^{2} \mathrm{sr}$ will saturate the detector. At the $2896 \mathrm{~K}$ molybdenum melting point, the spectral radiance due to Planck emission in-band is $4.7 \mathrm{~mW} / \mathrm{cm}^{2} \mathrm{sr}$, about three orders of magnitude greater than typical signals. Planck emission dominates wherever and whenever surfaces get hot enough, non-exclusively including leading edges of tiles and limiters after a disruption as shown in figure 3c, at surfaces impacted by a substantial flux of fast ions as discussed in section IV A, and at surfaces directly heated by absorbed RF power as discussed in section IV B.

\section{Bremsstrahlung emission}

Electrons scattering off of ions in hot plasmas emit a broadband continuum due to visible bremsstrahlung (VB), with the spectral radiance described by ${ }^{32}$

$$
B_{b r}(\lambda)=\left(\frac{e^{2}}{4 \pi \epsilon_{0}}\right)^{3} \frac{c g_{f f} \Delta \lambda}{\lambda^{2}} \int \frac{8 \pi n_{e}^{2} Z_{e f f}}{3 m_{e}^{2} c^{3}} \sqrt{\frac{2 m_{e}}{3 \pi T_{e}}} e^{-\frac{h c / \lambda}{T_{e}}} d \ell
$$

In typical C-Mod operating conditions, this emission is comparable to atomic emission for views looking through any portion of the plasma core as shown in figure 3a. When VB dominates other continuum sources, reflections are negligible, and the density and temperature are well diagnosed i.e. via Thomson scattering, a fan from the observed emission can be used as a diagnostic for the plasma effective charge state $Z_{\text {eff }}$ profile using an Abel inversion ${ }^{33}$. 


\section{Molecular emission}

Excitation of rotational and vibrational modes of molecular deuterium in the cold plasma boundary results in discrete line emission which has been well characterized ${ }^{34}$ and $\operatorname{modeled}^{35}$, and can be used to measure the molecular particle flux ${ }^{36}$. In the spectral region of interest, emission from several closely spaced molecular lines appears as a quasi-continuum. Volumetric molecular $D_{2}$ emission exceeds atomic Mo I emission only at high density $\left(n_{e}>10^{20} / \mathrm{m}^{3}\right)$ operation. In well boronized conditions when molybdenum emission is strongly suppressed, molecular emission contributes primarily near leading edges of limiters and views tangential to the inner wall and other PFC surfaces, as shown in figure 3b. Since molecular emission only appears at the highest densities and most camera views also look through the plasma core, VB emission generally dominates molecular emission.

\section{INITIAL RESULTS}

This new camera setup is now used to gain insight into the operation of radio frequency heating and current drive systems at Alcator C-Mod.

\section{A. Erosion and localized surface heating during Ion cyclotron heating}

Two ion-cyclotron range of frequency heating (ICRH) antenna systems exist at C-Mod. One is toroidally aligned $(\mathrm{TA})^{37}$ similar to installations at most other machines, which exerts a substantial component of electric field parallel to the magnetic field. Another is magnetic field aligned $(\mathrm{FA})^{38}$ in order to minimize the parallel component of the electric field. In the discharges discussed below $B_{T}=5.1 T$ and $I_{p}=1 M A$. The TA antennas were operated at $80 \mathrm{MHz}$ and the FA antennas were operated at $50 \mathrm{MHz}$, delivering a combination of hydrogen and helium-3 minority species heating and mode conversion heating depending on the electron temperature and density, and minority species density.

Two color erosion imaging data during ICRH operation is shown in figure 4, where the line and continuum filters were switched between two similar discharges. Frames from three times are shown: the ohmic phase, operation of the FA ICRH antennas, and simultaneous operation of both the FA and TA antennas. The scene is shown at each time as viewed through each filter separately, as well as the subtraction of the two images, representing 
isolated neutral molybdenum atomic line emission. In the subtracted images, color is mapped to an inferred molybdenum erosion flux divided by the $\mathrm{S} / \mathrm{XB}$ value: $\Gamma_{M o} /(\mathrm{S} / \mathrm{XB})$ since an image-wide measurement of the electron density and temperature was not possible.

In the ohmic phase, the continuum filtered image reveals a diffuse emission from the plasma core region associated with $\mathrm{VB}$, and a slight emission increase towards the active divertor interpreted as molecular deuterium emission. In the isolated line emission image, bright spots along the inner wall near the mid-plane and a stripe along the outer strike point in the divertor are the sole features in the image, consistent with the prior result that these regions represent the peak molybdenum emission during ohmic operation ${ }^{39}$.

During the FA ICRH phase, the continuum image is spatially similar to the ohmic phase. Continuum brightness is observed to increase, consistent with enhanced VB due to accumulation of eroded molybdenum into the plasma core during ICRH operation ${ }^{40}$. The isolated line emission image shows spatially localized emission increase compared to ohmic operation in a broad region along the upper shelf of the outer divertor and at the mid-plane of the main poloidal $(\mathrm{GH})$ limiter. Increased brightness is expected to occur due to a combination of increased power due to ICRH operation, and also fast ion impact ${ }^{41}$ on limiter surfaces.

During the combined FA and TA ICRH phase, a bright hot spot appears on the main limiter. Though both camera images saturate in the hot spot, a co-incident spectrometer view of the region observes a strong gradient continuum consistent with a black-body Planck spectrum for a $1615 \mathrm{~K}$ surface temperature, as shown in figure 5. Compared with the relatively unchanged divertor heat flux and temperature, these observations reveal the importance of monitoring the entire wall to avoid missing unexpected interaction points.

In each of the phases described above, inferring a sputtered molybdenum flux from the neutral molybdenum line brightness measured by the camera requires a separately measured electron temperature and density to specify the appropriate S/XB value. Since we do not have an imaging measurement of the electron temperature and density to match the camera data, we must use the fixed Langmuir probes which were described above, and from which relevant measurements are tabulated with their associated S/XB coefficients in table $\mathrm{I}$. The resulting maximum observed erosion flux values at the inner wall range from $\Gamma_{M o}=$ $5.5 \times 10^{14} / \mathrm{cm}^{2} s$ during ohmic operation, $2.7-6.4 \times 10^{15} / \mathrm{cm}^{2} s$ during FA ICRH, and $0.79-$ $1.9 \times 10^{14} / \mathrm{cm}^{2} s$ during FA and TA ICRH. At the outer divertor, maximum erosion fluxes ranged from $\Gamma_{M o}=2.7 \times 10^{15} / \mathrm{cm}^{2} s$ during ohmic operation, $5.2 \times 10^{15} / \mathrm{cm}^{2} s$ during $\mathrm{FA}$ 
ICRH, and $8.6 \times 10^{15} / \mathrm{cm}^{2} s$ during FA and TA ICRH.

Though the Mo I emission increased at the main limiter during ICRH operation, the ICRH interferes with mid-plane Langmuir probe measurements. The maximum erosion flux observed during ohmic operation was $\Gamma_{M o}=9.1 \times 10^{13} / \mathrm{cm}^{2} s$. The peak spectral line brightnesses at the main limiter increase from $I=6.1 \times 10^{12} / \mathrm{cm}^{2} \mathrm{sr} / \mathrm{s}$ during ohmic operation, to $1.5 \times 10^{13} / \mathrm{cm}^{2} \mathrm{sr} / \mathrm{s}$ during FA ICRH, to $4.6 \times 10^{13} / \mathrm{cm}^{2} \mathrm{sr} / \mathrm{s}$ during FA + TA ICRH. Since we have no electron temperature and density measurements during ICRH at the mid-plane, by assuming that the electron density and temperature increase or stay the same as in ohmic operation, the maximum erosion flux will also then increase. This apparent increase of erosion during ICRH operation is consistent with prior observations from C-Mod ${ }^{42}$ and $\mathrm{ASDEX}^{43}$. In addition to the increase of maximum erosion flux, the poloidal extent of erosion flux also appears to increase.

\begin{tabular}{ll||rrr} 
& \multicolumn{1}{c||}{ time } & $0.45 \mathrm{~s}$ & $0.85 \mathrm{~s}$ & $1.02 \mathrm{~s}$ \\
\hline \hline Inner wall & $n_{e}$ & $5 \times 10^{16}$ & $8 \times 10^{16}$ & $10^{17}$ \\
& $T_{e}$ & 20 & $30-50$ & $30-50$ \\
& $S / X B$ & 2.2 & $4.4-10.5$ & $4.4-10.5$ \\
\hline Main limiter* & $n_{e}$ & $10^{18}$ & - & - \\
& $T_{e}$ & 15 & - & - \\
& $S / X B$ & 1.2 & - & - \\
\hline Outer divertor & $n_{e}$ & $5 \times 10^{19}$ & $10^{20}$ & $10^{20}$ \\
& $T_{e}$ & 30 & 30 & 40 \\
& $S / X B$ & 6.0 & 8.0 & 13.0
\end{tabular}

TABLE I. Measurements of electron density in $m^{-3}$ and temperature in $e V$ taken by Langmuir probes in the various regions observed by the camera, taken at the same time as the frames in figure $4 . \mathrm{S} / \mathrm{XB}$ coefficients corresponding to these measurements from the ADAS file sxb93\#mo_llu\#mo0.dat are also shown. *Data from the main limiter was measured in a separate experiment under similar core conditions as those shown.

The mid-plane hot spot is also confirmed via thermocouples which reveal a peak of 
shot-integrated energy deposition on the limiter at the same location, as shown in figure 6. Dividing the shot integrated peak limiter energy deposition by the FA + TA ICRH time on, we obtain an average power deposition of $70 \mathrm{MW} / \mathrm{m}^{2}$. Energy deposited on the limiter empirically scales like fast ion loss, generally increasing as $P_{I C R H} / n_{e}$ increases (see figure 6), or $I_{p}$ decreases (not shown). While thermocouple and point-view spectroscopic measurements of this limiter heating previously existed, these new camera measurements contribute time and space resolved observations of the limiter heating. This reveals the toroidal and poloidal extent of limiter heating as shown in figure 4, which was not previously possible.

As shown in figure 5 for the two present shots comparing the time histories of line and continuum intensity measured from a point viewed by the spectrometer and camera, the two measurements generally agree within error bars. This example serves to prove the principle of separating line and continuum emission in an imaging diagnostic, but uncontrollable differences between the two sequential tokamak discharges contraindicate use of this technique for highly quantitative measurements unless simultaneous two-color imaging is used.

Fast ion impact at the mid-plane of the main limiter is believed to cause the observed hot spot during ICRH operation. ICRH is known to cause fast ion generation ${ }^{41}$, and modeling has revealed that fast ion orbits are elongated in the major radial direction ${ }^{44}$, which can intersect mid-plane limiters at sufficiently high energies. At Tore-Supra, a similar mid-plane surface heating associated with fast ions was observed ${ }^{9}$, though this phenomenon is distinct from hot spots on antenna limiters associated with rectified RF sheaths ${ }^{45}$.

\section{B. Localized surface heating during lower hybrid current drive}

Lower hybrid current drive (LHCD) is one potential non-inductive steady-state current drive technique for a tokamak ${ }^{16}$. Experiments at C-Mod have demonstrated strong single pass absorption of LH power and high total current drive fractions in scenarios with total current in the range $I_{p}=400-800 k A$, line averaged densities of $\bar{n}_{e} \sim 6-8 \times 10^{19} / \mathrm{m}^{3}$, and for LH power of $800 \mathrm{~kW}$ as evidenced by the low inductive loop voltage necessary to maintain current in the shots shown in figure 7. Bright spots appear on the main limiter during LHCD, consistent with Planck emission from hot spots observed by spectrometer views coincident with these locations in the camera view. Temperatures in the hot spots 
generally remain within the camera detection limits (i.e. $<1850 K$ ). The strongest hot spots are observed to move up the limiter as plasma current is increased, following a magnetic field line mapping of the four LH launcher waveguide rows to the main limiter almost on the opposite side of the tokamak, as indicated by the circles shown in figure 7 . Similar observations have been made at the JET, Tore-Supra ${ }^{17}$, and EAST ${ }^{18}$ tokamaks.

Several potential limiter heating mechanisms due to LHCD include direct heating by partially reflected RF power, fast electrons generated in the scrape-off layer (SOL), and poloidal electron density peaking, all of which are discussed below.

The RF ray-tracing code GENRAY ${ }^{46}$ was used to determine that the LH wave resonance cones do not intersect the limiter surfaces, as shown in 8. GENRAY modeling shows that the $15 \%$ of wave power launched in the co-current $\left(+n_{\|}\right)$direction absorbs via electron Landau damping after a $90^{\circ}$ toroidal transit, while the remaining $85 \%$ launched in the counter-current $\left(-n_{\|}\right)$direction propagates for many toroidal transits while damping on the electrons and does not exit the plasma along an alignment to the magnetic field. Several smaller hot-spots also observed on the limiter and lower divertor are possibly due to heating from $-n_{\|}$LH power scattered around the vessel, as suggested to occur by simulations ${ }^{47}$. These observations stand in contrast to observations of high-harmonic fast-wave heating at $\mathrm{NSTX}^{48}$, where RF power flows along magnetic field lines in the SOL.

Previous studies have suggested that a small fraction of LH power can generate fast electrons during the transit across the scrape-off layer (SOL) into the plasma core ${ }^{17,49}$. While such fast-electrons accelerated in the SOL could contribute to limiter heating, the low electron temperature in the SOL suggests that only a vanishingly small fraction of wave energy would accelerate electrons in this region, so limiter heating due to this mechanism is expected to be negligible ${ }^{50}$.

Previous studies at Alcator C-Mod have identified convective type electron density peaking in front of the LHCD waveguide rows $^{51,52}$. Such density peaking in the SOL would persist along magnetic field lines from the waveguides, and would result in a proportional increase of heat flux at the limiters, so this mechanism is believed to primarily cause the observed heat flux. 


\section{DISCUSSION}

Having identified these sources of heating and erosion during RF actuation, it is worth considering how they might be mitigated for future operations. In some cases, heat fluxes estimated to exceed $70 \mathrm{MW} / \mathrm{m}^{2}$ are incident on the limiter. While tolerable in a pulsed machine like C-Mod, heat flux this high would melt molybdenum or tungsten tiles in a steady state burning plasma experiment, where alpha particle impact with the limiter could cause even higher heat fluxes. Operating at high plasma current and density will help to reduce heat flux due to ICRH generated fast ions, but due to their higher overall energy compared to ICRH fast ions, fusion alpha orbits will be even more radially pronounced at the mid-plane and may require other mitigation approaches. In a larger reactor with similar fields, we would expect the ICRH orbit effects on PFCs to be less pronounced.

The fast ion heat flux to the main limiter is particularly exacerbated by the extremely limited toroidal extent and poloidal profile of the limiter. Building a more toroidally extended mid-plane limiter could also help to spread out heat flux if the fast ion sources cannot be otherwise mitigated. Either plasma shaping or limiter contouring could be attempted to reduce the observed poloidal peaking of the erosion and heat fluxes on the main limiter and inner wall. Where machine features must be placed close to the plasma, such as the ICRH and LHCD antennas, it may be preferable to install these above or below the mid-plane, or even on the inner wall, so that the fast-ion heat fluxes can be avoided.

While imaging diagnostics are powerful in their spatial coverage, they remain limited by some supporting measurements. For example, interpreting a particle erosion from surfaces across the full field of view requires electron density and temperature measurements also across the full field of view. Mapping of fixed Langmuir probe data to the camera view requires interpolation and an assumption of toroidal symmetry, which can be violated in the presence of modest error fields or other plasma boundary perturbations ${ }^{53}$. An imaging measurement of plasma temperature and density at surfaces would be more robust, and may be possible in future studies using helium line ratio techniques and appropriate interference filters $^{54,55}$.

In this study, use of the bright UV neutral molybdenum emission was prohibited by our imaging system design which uses a coherent imaging fiber bundle with weak transmission at UV wavelengths. A nuclear environment such as a burning plasma device will 
have neutron flux sufficient to rapidly reduce transmission even in the visible wavelength range in the glass ${ }^{24}$ used in such fiber bundles beyond the point of efficacy for plasma diagnosis. Though special low-impurity radiation hardened glasses can minimize this effect, implementing periscopes which use reflective optics such as those at JET ${ }^{56}$ and DIII-D ${ }^{57}$ is probably a better long term solution.

The technique of switching filters between similar shots is extremely limiting in the wavelength space which can be inspected, and is susceptible to substantial error due to minor variations between subsequent shots. Simultaneous imaging of multiple spectral bands is desirable, especially for a realtime diagnostic, and could be realized with image splitting techniques. Using an appropriate set of image splitters, light recorded from line emission and nearby continuum of neutral molybdenum and appropriate helium lines could produce the necessary data for imaging diagnostics of electron temperature and density, molybdenum erosion, surface temperature, and even effective charge state profiles from visible bremsstrahlung. This and other ${ }^{56}$ systems of multiplexed diagnostics will also help minimize the needed diagnostic port space, and maximize neutron blanket area.

\section{ACKNOWLEDGMENTS}

This work was supported by the US Department of Energy under contracts DE-AC5207NA27344 and DE-FC02-99ER54512, and in part by an appointment to the US DOE Fusion Energy Postdoctoral Research Program administered by ORISE. The authors would like to acknowledge support with hardware installation from the MIT Plasma Science and Fusion Center group and technicians, especially Gary Dekow, Rui Vieira, Andy Pfeiffer, and Maria Silveira; and the entire C-Mod team for operation of the experiment.

\section{Appendix A: Camera system design}

The camera system is similar to those previously deployed at DIII-D ${ }^{58,59}$, with some alterations. As shown in figure 9, light exits C-Mod through a glass window to a re-entrant periscope port and enters a Marshall 4306.5-1.5 $F 0_{\text {back }}=6.5 \mathrm{~mm} \mathrm{f} / 1.5$ miniature lens with focus set to $F 0_{\text {front }} \sim 1 \mathrm{~m}$, mounted to a 3 meter Schott IG-154 coherent imaging fiber bundle. Before the 2012 experimental campaign, the bundle transmission at 550nm was 
measured to be 0.5 and after the campaign it had reduced to 0.3 due most likely to radiation damage $^{24}$. After routing through the bundle, the image projects through a Computar V2514 $F 1_{\text {back }}=25 \mathrm{~mm} f / 1.31$ inch C-mount format lens with focus set to $F 1_{\text {front }}=\infty$. A custom interference filter selects a narrow spectral band of interest, which is then received by a Fujinon CF50B $F 2_{\text {back }}=50 \mathrm{~mm} f / 1.41$ inch C-mount lens with focus set to $F 2_{\text {front }}=\infty$. In the CIDTEC I3710DX9 camera, light is amplified by an integrated Photonis XX1450UV 2nd generation hybrid intensifier with an S-20 photocathode and a P43 phosphor, which finally illuminates the CID sensor. Total effective throughput (etendue) per pixel is approximately $1.5 \times 10^{-6} \mathrm{~cm}^{2} \mathrm{sr}$.

The final image size projected on the sensor is determined by the magnification of the two lenses and the fiber taper as

$$
M=\frac{F 2_{\text {back }}}{F 1_{\text {back }}} M_{\text {taper }}
$$

For the present system with $F 1_{\text {back }}=25 \mathrm{~mm}, F 2_{\text {back }}=50 \mathrm{~mm}$, and $M_{\text {taper }}=5 / 8$, the effective magnification is $M=5 / 4$. This produces a projected image height of $5 \mathrm{~mm}$, which occupies roughly $83 \%$ of the full sensor height.

The interference filter transmission wavelength band is shifted slightly due to an effective angle of incidence $\theta=\operatorname{atan}\left(r / F 1_{b a c k}\right)$ for pixels away from the optical axis according to

$$
\Delta \lambda=\lambda_{0}\left(1-\sqrt{1-\frac{n_{0}^{2}}{n_{f}^{2}} \sin ^{2} \theta}\right)
$$

For the present system: the normal filter transmission center wavelength $\lambda_{0}=550.7 \mathrm{~nm}$, filter index of refraction $n_{f}=2.05$, air index $n_{0}=1$, back focal length $F 1_{\text {back }}=25 \mathrm{~mm}$, and the furthest pixel distance orthogonal to the optical axis $r=2.83 \mathrm{~mm}$, the wavelength shift is $\Delta \lambda=0.81 \mathrm{~nm}$. This shift may be reduced if desired by choosing a lens with a larger back focal length $F 1_{\text {back }}$.

\section{Appendix B: Empirical S/XB determination}

The $\mathrm{S} / \mathrm{XB}$ coefficient is determined by the ratio of ionization $(\mathrm{S})$ to excitation $(\mathrm{X})$ rates for a particular excited state divided by the branching ratio (B) for a specific transition

from that state, which are specific to the atomic emission observed, and generally a strong function of electron density and temperature. The S/XB values shown in figure 10 are not 
resolved in the quantum number $J$ representing the total angular momentum of the electron, so the effective value for a single line of the triplet must be calculated as

$$
(S / X B)_{e f f}=(S / X B) \frac{(2 S+1)(2 L+1)}{(2 J+1)}
$$

where the multiplicative factor has been tabulated in table II for the 550nm Mo I triplet. Note that the statistical weights are appropriately normalized.

$$
\sum_{J} \frac{(2 J+1)}{(2 S+1)(2 L+1)}=1
$$

\begin{tabular}{ccccccc}
$\lambda(\mathrm{nm})$ & upper level lower level $L$ & $S$ & $\frac{(2 S+1)(2 L+1)}{2 J+1}$ \\
\hline 550.649 & $z^{5} P_{1}^{0}$ & $a^{5} S_{2}^{0}$ & 1 & 2 & 3 & $15 / 7$ \\
553.305 & $z^{5} P_{2}^{0}$ & $a^{5} S_{2}^{0}$ & 1 & 2 & 2 & $15 / 5$ \\
557.045 & $z^{5} P_{3}^{0}$ & $a^{5} S_{2}^{0}$ & 1 & 2 & 1 & $15 / 3$
\end{tabular}

TABLE II. This table shows atomic configuration data relevant to the 550nm Mo I triplet lines ${ }^{61,62}$.

Accounting for the statistical weight, the average energy per photon, and assuming typical electron density and temperature $\left(n_{e}=5 \times 10^{19} / \mathrm{m}^{3}, T_{e}=10 \mathrm{eV}\right)$, the associated $\mathrm{S} / \mathrm{XB}$ value from ADAS is about 4.2. Typical camera settings then correspond to a molybdenum erosion flux sensitivity in the range of $\Gamma_{M o}=1.2 \times 10^{13}-3.0 \times 10^{15} / \mathrm{cm}^{2} / \mathrm{s}$.

Calculated S/XB coefficients from the Atomic Data and Analysis Structure (ADAS) have known discrepancies with experimentally measured values exceeding an order of magnitude ${ }^{15,60}$ for the $550.6 \mathrm{~nm}$ line of interest. Atomic modeling of the S/XB for ground state $n=1$ transitions, such as the $390.2 \mathrm{~nm}$ Mo I emission, is much more accurate and consistent with experiments than for higher $n$, potentially metastable transitions, such as the 550.6nm Mo I emission. For this reason, an empirical coefficient for the $550.6 \mathrm{~nm}$ line is desired.

At C-Mod, both the $390.2 \mathrm{~nm}$ and 550.6nm molybdenum emissions were observed near the outer divertor strike point in sequential experiments with similar operating conditions using the Chromex spectrometer system. A fixed Langmuir probe at roughly the same poloidal location, but toroidally distant, was used to measure the electron temperature and density. By assuming that the molybdenum erosion flux is identical in both experiments shown in figure 11, the following expression is derived:

$$
(S / X B)_{550.6 n m}=(S / X B)_{390.2 n m} \frac{I_{390.2 n m}}{I_{550.6 n m}}
$$


Substituting the better validated $390.2 \mathrm{~nm}$ S/XB from ADAS, and the experimentally determined line intensity ratio over a range of densities and temperatures observed at C-Mod (from figure 11), the empirical $\mathrm{S} / \mathrm{XB}$ is determined as shown in figure 10. The measured electron density and temperature are low-pass filtered to match the $10.8 \mathrm{~ms}$ spectrometer integration time. The resulting empirical $\mathrm{S} / \mathrm{XB}$ values have a similar discrepancy with ADAS values as prior studies at low temperature and density, but tend toward agreement with ADAS at higher temperatures.

\section{REFERENCES}

${ }^{1}$ A. G. McLean, Quantification of Chemical Erosion in the Divertor of the DIII-D Tokamak, Ph.D. thesis, University of Toronto (2010).

${ }^{2}$ V. Martin, V. Moncada, G. Dunand, Y. Corre, E. Delchambre, and J. Travere, "Integrated software for imaging data analysis applied to edge plasma physic and operational safety," Fusion Engineering and Design, 86, 270 (2011), ISSN 0920-3796.

${ }^{3}$ J.-M. Travere, "In-vessel components imaging systems: From the present experience towards ITER safe operation," Fusion Engineering and Design, 84, 1862 (2009), ISSN 0920-3796, proceeding of the 25th Symposium on Fusion Technology (SOFT-25).

${ }^{4} \mathrm{E}$. Gauthier, "Progress in diagnostics for characterization of plasma wall interaction in tokamaks," Journal of Nuclear Materials, 390-391, 1059 (2009), ISSN 0022-3115, proceedings of the 18th International Conference on Plasma-Surface Interactions in Controlled Fusion Devices.

${ }^{5} \mathrm{E}$. Tsitrone, "Key plasma wall interaction issues towards steady state operation," Journal of Nuclear Materials, 363-365, 12 (2007), ISSN 0022-3115, plasma-Surface Interactions17.

${ }^{6}$ J. Roth, E. Tsitrone, A. Loarte, T. Loarer, G. Counsell, R. Neu, V. Philipps, S. Brezinsek, M. Lehnen, P. Coad, C. Grisolia, K. Schmid, K. Krieger, A. Kallenbach, B. Lipschultz, R. Doerner, R. Causey, V. Alimov, W. Shu, O. Ogorodnikova, A. Kirschner, G. Federici, and A. Kukushkin, "Recent analysis of key plasma wall interactions issues for ITER," Journal of Nuclear Materials, 390-391, 1 (2009), ISSN 0022-3115, proceedings of the 18th International Conference on Plasma-Surface Interactions in Controlled Fusion Devices.

${ }^{7}$ M. Rubel, "Overview of Erosion-Deposition Diagnostic Tools for the ITER-Like Wall in 
the JET Tokamak," Proceedings of the 20th International Conference on Plasma Surface Interactions, EFDA-JET-CP(12)02/39 (2012).

${ }^{8}$ B. Unterberg, "Plasma-Wall Interaction Issues in DEMO," Proceedings of the Proceedings of the 20th International Conference on Plasma Surface Interactions (2012).

${ }^{9}$ P. Moreau, O. Barana, S. Brémond, L. Colas, A. Ekedahl, F. Saint-Laurent, C. Balorin, G. Caulier, C. Desgranges, D. Guilhem, M. Jouve, F. Kazarian, G. Lombard, L. Millon, R. Mitteau, P. Mollard, H. Roche, and J. Travere, "RF heating optimization on Tore Supra using feedback control of infrared measurements," Fusion Engineering and Design, 82, 1030 (2007), ISSN 0920-3796, proceedings of the 24th Symposium on Fusion Technology (SOFT-24).

${ }^{10}$ A. Loarte, B. Lipschultz, A. Kukushkin, G. Matthews, P. Stangeby, N. Asakura, G. Counsell, G. Federici, A. Kallenbach, K. Krieger, A. Mahdavi, V. Philipps, D. Reiter, J. Roth, J. Strachan, D. Whyte, R. Doerner, T. Eich, W. Fundamenski, A. Herrmann, M. Fenstermacher, P. Ghendrih, M. Groth, A. Kirschner, S. Konoshima, B. LaBombard, P. Lang, A. Leonard, P. Monier-Garbet, R. Neu, H. Pacher, B. Pegourie, R. Pitts, S. Takamura, J. Terry, E. Tsitrone, the ITPA Scrape-off Layer, and D. P. T. Group, "Chapter 4: Power and particle control," Nuclear Fusion, 47, S203 (2007).

${ }^{11}$ G. J. van Rooij, "Tungsten divertor erosion in all metal devices: lessons from the ITER-like wall of JET and the all tungsten ASDEX Upgrade," Proceedings of the 20th International Conference on Plasma Surface Interactions (2012).

${ }^{12}$ P. C. Stangeby, "An Experimental Comparison of Gross and Net Erosion of Mo in the DIII-D Divertor," Proceedings of the 20th International Conference on Plasma Surface Interactions (2012).

${ }^{13}$ N. H. Brooks, "Spectroscopic measurements of controlled molybdenum erosion in the DIIID divertor," Proceedings of the 20th International Conference on Plasma Surface Interactions (2012).

${ }^{14}$ R. A. Pitts, "A full tungsten divertor for ITER: physics issues and design status," Proceedings of the 20th International Conference on Plasma Surface Interactions (2012).

${ }^{15}$ D. Nishijima, R. P. Doerner, D. G. Whyte, M. J. Baldwin, and T. Schwarz-Selinger, "Measurements of Mo I S/XB values," Journal of Physics B: Atomic, Molecular and Optical Physics, 43, 225701 (2010).

${ }^{16}$ C. Gormezano, A. Sips, T. Luce, S. Ide, A. Becoulet, X. Litaudon, A. Isayama, J. Hobirk, 
M. Wade, T. Oikawa, R. Prater, A. Zvonkov, B. Lloyd, T. Suzuki, E. Barbato, P. Bonoli, C. Phillips, V. Vdovin, E. Joffrin, T. Casper, J. Ferron, D. Mazon, D. Moreau, R. Bundy, C. Kessel, A. Fukuyama, N. Hayashi, F. Imbeaux, M. Murakami, A. Polevoi, and H. S. John, "Chapter 6: Steady state operation," Nuclear Fusion, 47, S285 (2007).

${ }^{17}$ V. Petrzilka, V. Fuchs, J. Gunn, N. Fedorczak, A. Ekedahl, M. Goniche, J. Hillairet, and P. Pavlo, "Theory of fast particle generation in front of lh grills," Plasma Physics and Controlled Fusion, 53, 054016 (2011).

${ }^{18}$ K. Gan, M. Li, F. Wang, X. Gong, and X. Zhang, "Hot spots generated by low hybrid wave absorption in the SOL on the EAST tokamak," Journal of Nuclear Materials, (2013), ISSN 0022-3115.

${ }^{19}$ I. Hutchinson, "The Physics and Engineering of Alcator C-MOD," PFC Report, RR-8811 (1988).

${ }^{20}$ E. S. Marmar and Alcator C-Mod Group, "The alcator-c-mod program," Fusion Science and Technology, 51, 261 (2007).

${ }^{21}$ M. Greenwald, "Overview of experimental results and code validation activities at Alcator C-Mod," in IAEA Conference Proceedings, OV/2-3 (2012).

${ }^{22}$ B. LaBombard, J. Terry, J. Hughes, D. Brunner, J. Payne, M. Reinke, Y. Lin, and S. Wukitch, "Divertor heat flux footprints in EDA H-mode discharges on Alcator C-Mod," Journal of Nuclear Materials, 415, S349 (2011), ISSN 0022-3115, proceedings of the 19th International Conference on Plasma-Surface Interactions in Controlled Fusion.

${ }^{23}$ T. E. Evans, R. K. W. Roeder, J. A. Carter, and B. I. Rapoport, "Homoclinic tangles, bifurcations and edge stochasticity in diverted tokamaks," Contributions to Plasma Physics, 44, 235 (2004), ISSN 1521-3986.

${ }^{24}$ C. P. Chrobak, M. A. V. Zeeland, R. A. Moyer, and J. H. Yu, "Restoring transmission of irradiated image fiber bundles," Review of Scientific Instruments, 83, 10E514 (2012).

${ }^{25}$ D. L. Rudakov, J. A. Boedo, R. A. Moyer, A. Litnovsky, V. Philipps, P. Wienhold, S. L. Allen, M. E. Fenstermacher, M. Groth, C. J. Lasnier, R. L. Boivin, N. H. Brooks, A. W. Leonard, W. P. West, C. P. C. Wong, A. G. McLean, P. C. Stangeby, G. D. Temmerman, W. R. Wampler, and J. G. Watkins, "First tests of molybdenum mirrors for ITER diagnostics in DIII-D divertor," Review of Scientific Instruments, 77, 10F126 (2006).

${ }^{26}$ G. J. van Rooij, "Characterization of tungsten sputtering in the JET divertor," in IAEA Conference Proceedings, EX/P5-05 (2012). 
${ }^{27}$ M. L. Reinke, Y. A. Podpaly, M. Bitter, I. H. Hutchinson, J. E. Rice, L. Delgado-Aparicio, C. Gao, M. Greenwald, K. Hill, N. T. Howard, A. Hubbard, J. W. Hughes, N. Pablant, A. E. White, and S. M. Wolfe, "X-ray imaging crystal spectroscopy for use in plasma transport research," Review of Scientific Instruments, 83, 113504 (2012).

${ }^{28}$ K. Behringer, H. P. Summers, B. Denne, M. Forrest, and M. Stamp, "Spectroscopic determination of impurity influx from localized surfaces," Plasma Physics and Controlled Fusion, 31, 2059 (1989).

${ }^{29}$ D. N. Hill, R. Ellis, W. Ferguson, D. E. Perkins, T. Petrie, and C. Baxi, "Infrared thermography of the diii-d divertor targets," Review of Scientific Instruments, 59, 1878 (1988).

${ }^{30}$ M.-H. Aumeunier, M. Firdaouss, J.-M. Travere, T. Loarer, E. Gauthier, V. Martin, D. Chabaud, E. Humbert, and J.-E. Contributors, "Modeling of the iter-like wide-angle infrared thermography view of jet," Review of Scientific Instruments, 83, 10D522 (2012).

${ }^{31}$ J. L. Terry, B. LaBombard, D. Brunner, J. Payne, and G. A. Wurden, "Divertor ir thermography on alcator c-mod," Review of Scientific Instruments, 81, 10E513 (2010).

${ }^{32}$ I. Hutchinson, Principles of Plasma Diagnostics (Cambridge University Press, 2002).

${ }^{33}$ D. P. Schissel, R. E. Stockdale, H. S. John, and W. M. Tang, "Measurements and implications of z[sub eff] profiles on the diii-d tokamak," Physics of Fluids, 31, 3738 (1988).

${ }^{34}$ R. S. Freund, J. A. Schiavone, and H. M. Crosswhite, "The electronic spectrum and energy levels of the deuterium molecule," Journal of Physical and Chemical Reference Data, 14, 235 (1985).

${ }^{35}$ U. Fantz, B. Schalk, and K. Behringer, "Calculation and interpretation of the continuum radiation of hydrogen molecules," New Journal of Physics, 2, 7 (2000).

${ }^{36}$ E. M. Hollmann, S. Brezinsek, N. H. Brooks, M. Groth, A. G. McLean, A. Y. Pigarov, and D. L. Rudakov, "Spectroscopic measurement of atomic and molecular deuterium fluxes in the diii-d plasma edge," Plasma Physics and Controlled Fusion, 48, 1165 (2006).

${ }^{37} \mathrm{P}$. T. Bonoli, "Wave-particle studies in the ion cyclotron and lower hybrid ranges of frequencies in alcator c-mod," Fusion Science and Technology, 51, 401 (2007).

${ }^{38}$ M. Garrett and S. Wukitch, "Mitigation of radio frequency sheaths through magnetic fieldaligned ICRF antenna design," Fusion Engineering and Design, 87, 1570 (2012), ISSN 0920-3796.

${ }^{39}$ D. Pappas, Study of Molybdenum sources and screening in the Alcator C-Mod tokamak, 
Ph.D. thesis, Massachusetts Institute of Technology, Cambridge, MA 02139 USA (2000).

${ }^{40}$ B. Lipschultz, D. Pappas, B. LaBombard, J. Rice, D. Smith, and S. Wukitch, "Molybdenum sources and transport in Alcator C-Mod," Journal of Nuclear Materials, 290-293, 286 (2001), ISSN 0022-3115, 14th Int. Conf. on Plasma-Surface Interactions in Controlled Fusion Devices.

${ }^{41}$ D. C. Pace, R. S. Granetz, R. Vieira, A. Bader, J. Bosco, D. S. Darrow, C. Fiore, J. Irby, R. R. Parker, W. Parkin, M. L. Reinke, J. L. Terry, S. M. Wolfe, S. J. Wukitch, and S. J. Zweben, "Energetic ion loss detector on the Alcator C-Mod tokamak," Review of Scientific Instruments, 83, 073501 (2012).

${ }^{42}$ B. Lipschultz, D. Pappas, B. LaBombard, J. Rice, D. Smith, and S. Wukitch, "A study of molybdenum influxes and transport in alcator c-mod," Nuclear Fusion, 41, 585 (2001).

${ }^{43}$ R. Dux, V. Bobkov, N. Fedorczak, K. Iraschko, A. Kallenbach, R. Neu, T. Pütterich, and V. Rohde, "Tungsten erosion at the ICRH limiters in ASDEX Upgrade," Journal of Nuclear Materials, 363-365, 112 (2007), ISSN 0022-3115, ¡ce:title¿Plasma-Surface Interactions- $17 \mathrm{i} /$ ce:title $_{i}$.

${ }^{44}$ G. J. Kramer, R. V. Budny, A. Bortolon, E. D. Fredrickson, G. Y. Fu, W. W. Heidbrink, R. Nazikian, E. Valeo, and M. A. V. Zeeland, "A description of the full-particle-orbitfollowing spiral code for simulating fast-ion experiments in tokamaks," Plasma Physics and Controlled Fusion, 55, 025013 (2013).

${ }^{45}$ L. Colas, L. Costanzo, C. Desgranges, S. Brémond, J. Bucalossi, G. Agarici, V. Basiuk, B. Beaumont, A. Bécoulet, and F. Nguyen, "Hot spot phenomena on tore supra icrf antennas investigated by optical diagnostics," Nuclear Fusion, 43, 1 (2003).

${ }^{46}$ A. P. Smirnov, R. W. Harvey, and K. Kupfer, "A general ray tracing code genray," in Bull. Am. Phys. Soc. (1994).

${ }^{47}$ S. Shiraiwa, J. Ko, O. Meneghini, R. Parker, A. E. Schmidt, S. Scott, M. Greenwald, A. E. Hubbard, J. Hughes, Y. Ma, Y. Podpaly, J. E. Rice, G. Wallace, J. R. Wilson, S. M. Wolfe, and A. C.-M. Group, "Full wave effects on the lower hybrid wave spectrum and driven current profile in tokamak plasmas," Physics of Plasmas, 18, 080705 (2011).

${ }^{48}$ R. J. Perkins, J. C. Hosea, G. J. Kramer, J.-W. Ahn, R. E. Bell, A. Diallo, S. Gerhardt, T. K. Gray, D. L. Green, E. F. Jaeger, M. A. Jaworski, B. P. LeBlanc, A. McLean, R. Maingi, C. K. Phillips, L. Roquemore, P. M. Ryan, S. Sabbagh, G. Taylor, and J. R. Wilson, "High-Harmonic Fast-Wave power flow along magnetic field lines in the Scrape-Off 
Layer of NSTX," Phys. Rev. Lett., 109, 045001 (2012).

${ }^{49}$ M. Goniche, J. Mailloux, Y. Demers, V. Fuchs, P. Jacquet, C. Boucher, A. Côté, C. Côté, J. Gunn, B. Terreault, P. Bibet, P. Froissard, D. Guilhem, J. Harris, G. Rey, and M. Tareb, "Strong toroidal asymmetries in power deposition on divertor and first wall components during LHCD on TdeV and Tore Supra," Journal of Nuclear Materials, 241-243, 745 (1997), ISSN 0022-3115.

${ }^{50}$ G. M. Wallace, Behavior of Lower Hybrid Waves in the Scrape Off Layer of a Diverted Tokamak, Ph.D. thesis, Massachusetts Institute of Technology, Cambridge, MA 02139 USA (2010).

${ }^{51}$ C. Lau, G. R. Hanson, B. Labombard, Y. Lin, O. Meneghini, R. Ochoukov, R. Parker, S. Shiraiwa, J. Terry, G. Wallace, J. Wilgen, and S. J. Wukitch, "Effects of LH power on SOL density profiles and LH coupling on Alcator C-Mod," Plasma Physics and Controlled Fusion, 55, 025008 (2013).

${ }^{52} \mathrm{C}$. Lau, Effects of RF power on $S O L$ density profiles and RF coupling on the Alcator $C$ Mod Tokamak, Ph.D. thesis, Massachusetts Institute of Technology, Cambridge, MA 02139 USA (2013).

${ }^{53}$ D. Harting, Y. Liang, S. Jachmich, R. Koslowski, G. Arnoux, S. Devaux, T. Eich, E. Nardon, D. Reiter, H. Thomsen, and J. E. contributors, "Strike point splitting in the heat and particle flux profiles compared with the edge magnetic topology in a $n=2$ resonant magnetic perturbation field at JET," Nuclear Fusion, 52, 054009 (2012).

${ }^{54}$ C. S. Pitcher, B. Labombard, B. Lipschultz, J. L. Terry, R. E. Bell, and S. J. Zweben, "Edge Measurements on Alcator C-Mod using the Helium Line Ratio Technique," in APS Meeting Abstracts (2000) p. 1110P.

${ }^{55}$ E. A. Unterberg, O. Schmitz, D. H. Fehling, H. Stoschus, C. C. Klepper, J. M. MunozBurgos, G. V. Wassenhove, and D. L. Hillis, "HELIOS: A helium line-ratio spectralmonitoring diagnostic used to generate high resolution profiles near the ion cyclotron resonant heating antenna on TEXTOR," Review of Scientific Instruments, 83, 10D722 (2012).

${ }^{56}$ A. Huber, S. Brezinsek, P. Mertens, B. Schweer, G. Sergienko, A. Terra, G. Arnoux, N. Balshaw, M. Clever, T. Edlingdon, S. Egner, J. Farthing, M. Hartl, L. Horton, D. Kampf, J. Klammer, H. T. Lambertz, G. F. Matthews, C. Morlock, A. Murari, M. Reindl, V. Riccardo, U. Samm, S. Sanders, M. Stamp, J. Williams, K. D. Zastrow, C. Zauner, and J.-E. 
Contributors, "Development of a mirror-based endoscope for divertor spectroscopy on jet with the new iter-like wall (invited)," Review of Scientific Instruments, 83, 10D511 (2012). ${ }^{57}$ M. Fenstermacher, X. Xu, I. Joseph, M. Lanctot, C. Lasnier, W. Meyer, B. Tobias, L. Zeng, A. Leonard, and T. Osborne, "Fast pedestal, $\{\mathrm{SOL}\}$ and divertor measurements from diii-d to validate bout++ nonlinear $\{$ ELM $\}$ simulations," Journal of Nuclear Materials, (2013), ISSN 0022-3115.

${ }^{58}$ M. Groth, R. M. Ellis, N. H. Brooks, M. E. Fenstermacher, C. J. Lasnier, W. H. Meyer, and J. M. Moeller, "Measurements of spatial line emission profiles in the main scrape-off layer of the DIII-D tokamak," Review of Scientific Instruments, 80, 033505 (2009).

${ }^{59}$ M. E. Fenstermacher, W. H. Meyer, R. D. Wood, D. G. Nilson, R. Ellis, and N. H. Brooks, "A tangentially viewing visible TV system for the DIII-D divertor," Review of Scientific Instruments, 68, 974 (1997), ISSN 00346748.

${ }^{60}$ R. P. Doerner, D. G. Whyte, and D. M. Goebel, "Sputtering yield measurements during low energy xenon plasma bombardment," Journal of Applied Physics, 93, 5816 (2003).

${ }^{61}$ J. Sugar and A. Musgrove, "Energy levels of molybdenum, mo i through mo xlii," Journal of Physical and Chemical Reference Data, 17, 155 (1988).

${ }^{62} \mathrm{~W}$. Whaling and J. W. Brault, "Comprehensive transition probabilities in mo i," Physica Scripta, 38, 707 (1988). 


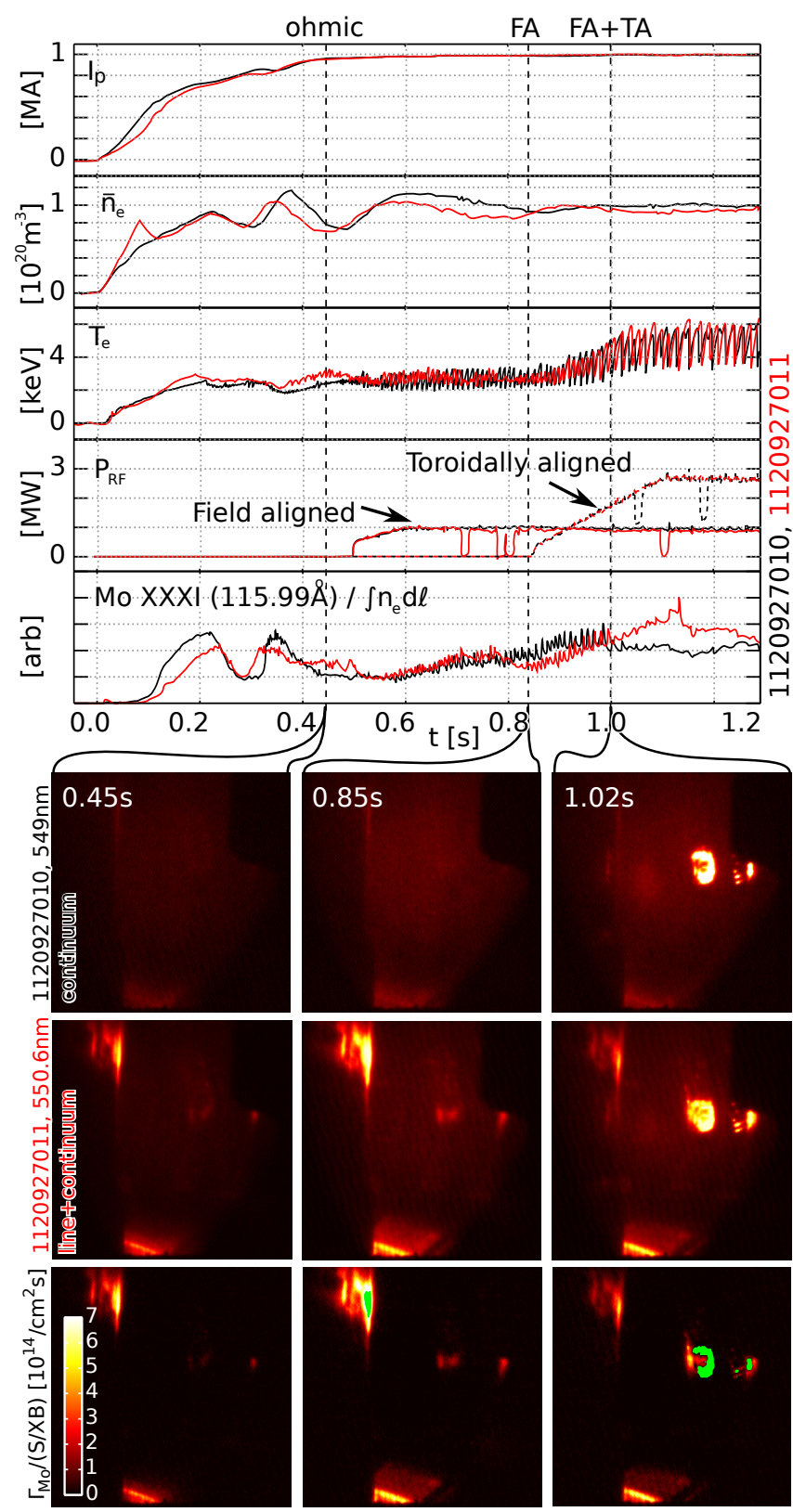

FIG. 4. Above: Plasma current, line averaged electron density, core electron temperature, ICRH heating power (FA solid, TA dashed), and core molybdenum content for two shots in which the line and continuum filters were used. Below: Images from the filters and their subtraction, scaled to represent particle flux divided by S/XB during three operation phases: ohmic heating, FA ICRH, and FA+TA ICRH. Regions of saturation are outlined in green in the subtracted image. 


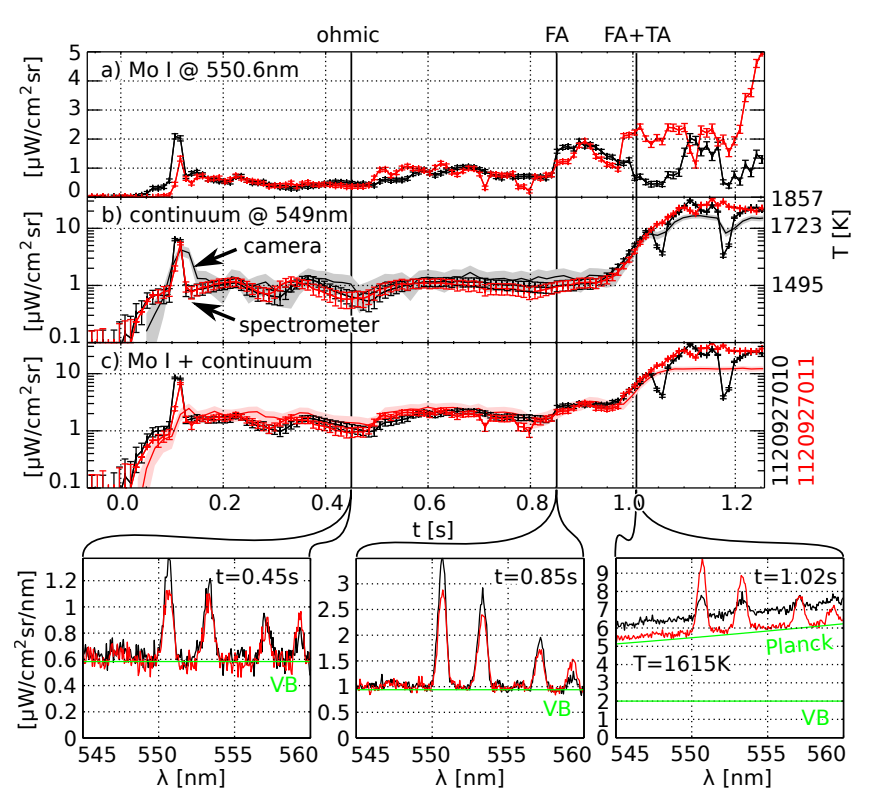

FIG. 5. Above: Time histories from the middle of the main limiter (circled in white in figure 1) of spectrometer (lines with error-bars) and camera data (lines with colored shaded error bands in panels b and c) for a) fitted line emission intensity, b) continuum intensity, and c) their sum integrated over the camera filter bands. Error bars shown are calculated from photon statistics. Below: Example spectra are shown at the times of the three camera frames in figure 4. Continuum contributions are shown in green.
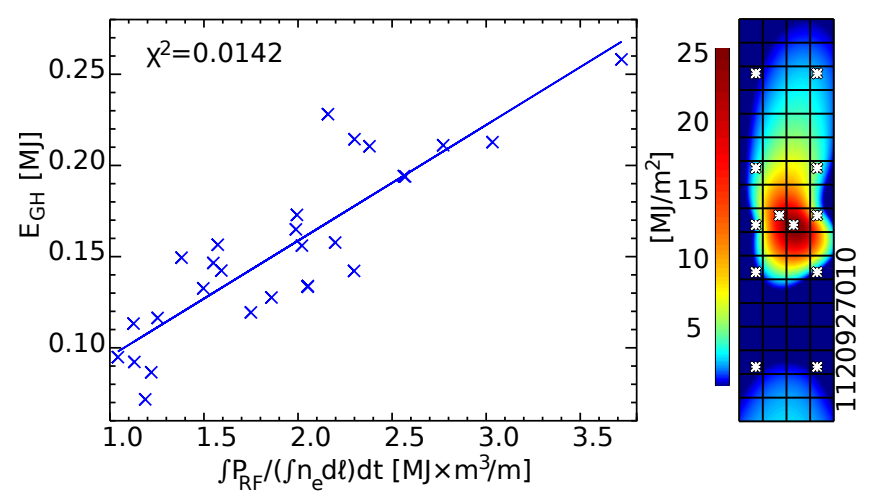

FIG. 6. Left: Total energy deposited on the main limiter plotted vs. shot-integrated ICRH power divided by electron density, for all shots on a run-day. A fitted line and the unreduced chi-squared goodness of fit parameter are also shown. Right: Energy deposition on the main limiter averaged over a shot. Thermocouple locations are marked by a white asterisk, and energy deposition is interpolated across the limiter face by a $2 \mathrm{D}$ spline. 

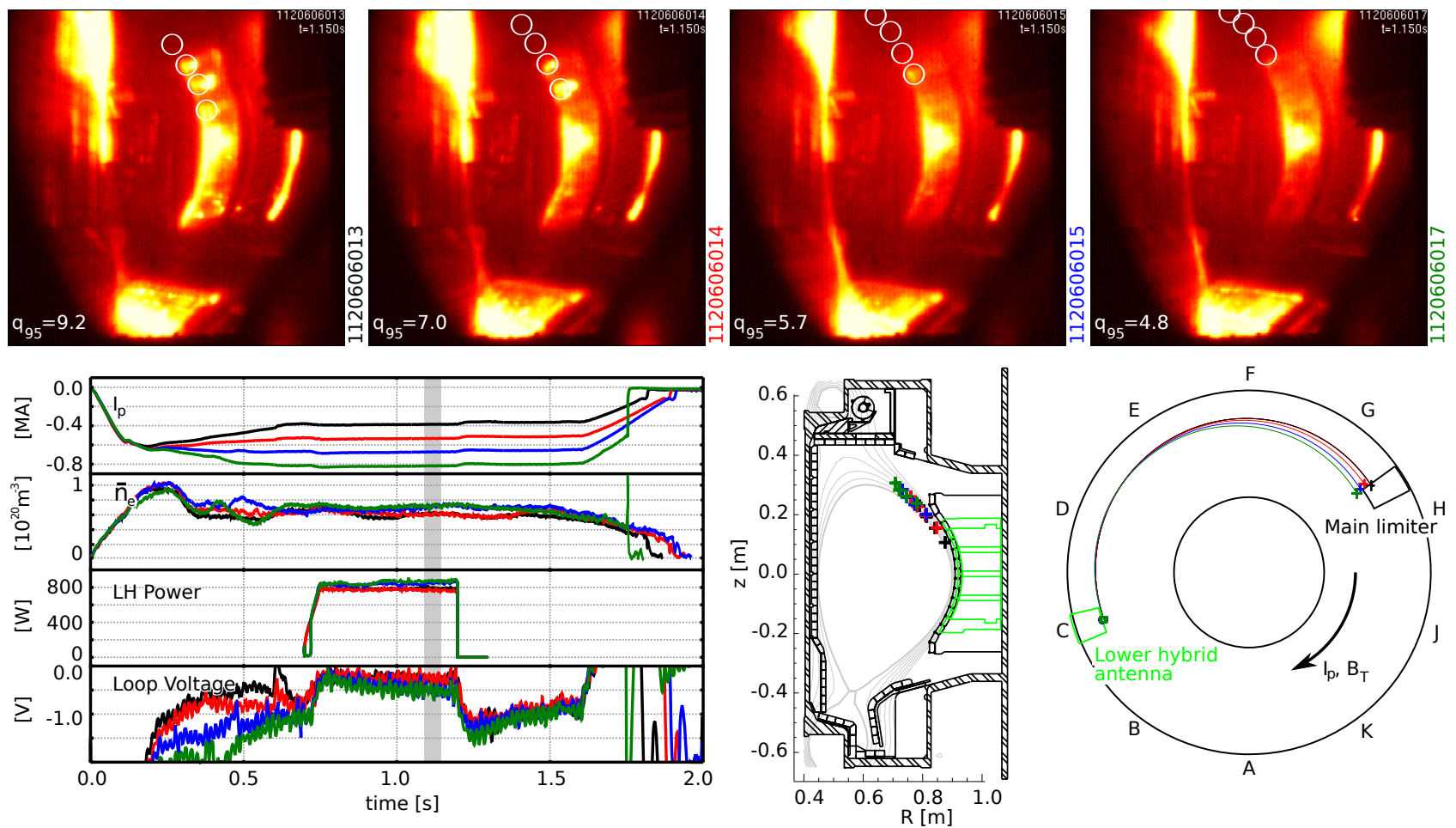

FIG. 7. Top: Camera data from the 550.6nm filter with magnetic field line mapping to the toroidal location of the limiter edge circled. Lower left: Plasma current, line integrated electron density, LH power, and inductive loop voltage for these experiments. Lower right: Field line tracing connecting the LH waveguides to the main limiter.

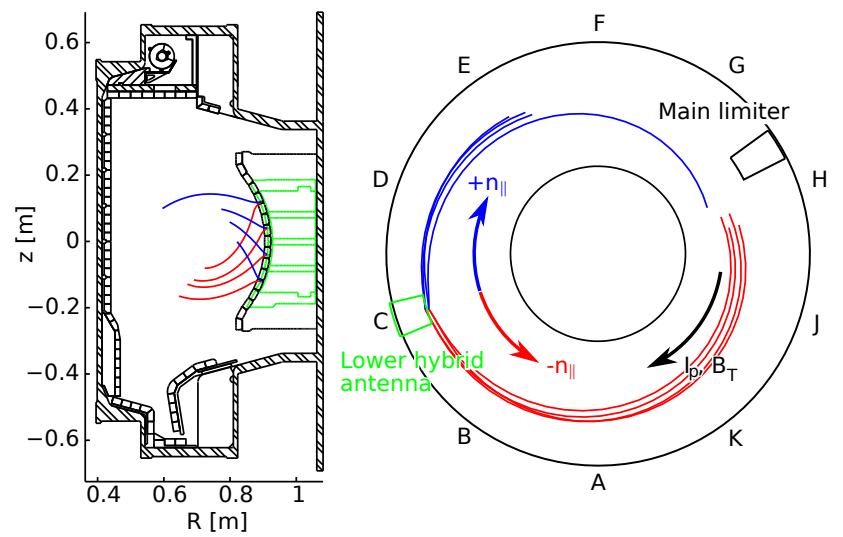

FIG. 8. Ray-mapping of LH waves from the code GENRAY illustrating that launched waves penetrate into the plasma core as opposed to propagating in the boundary. 


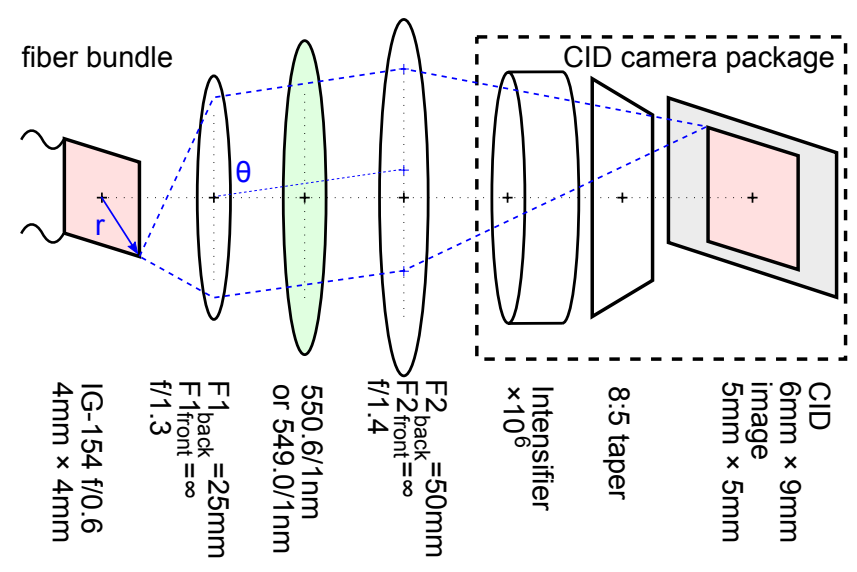

FIG. 9. A schematic of the imaging optics layout.
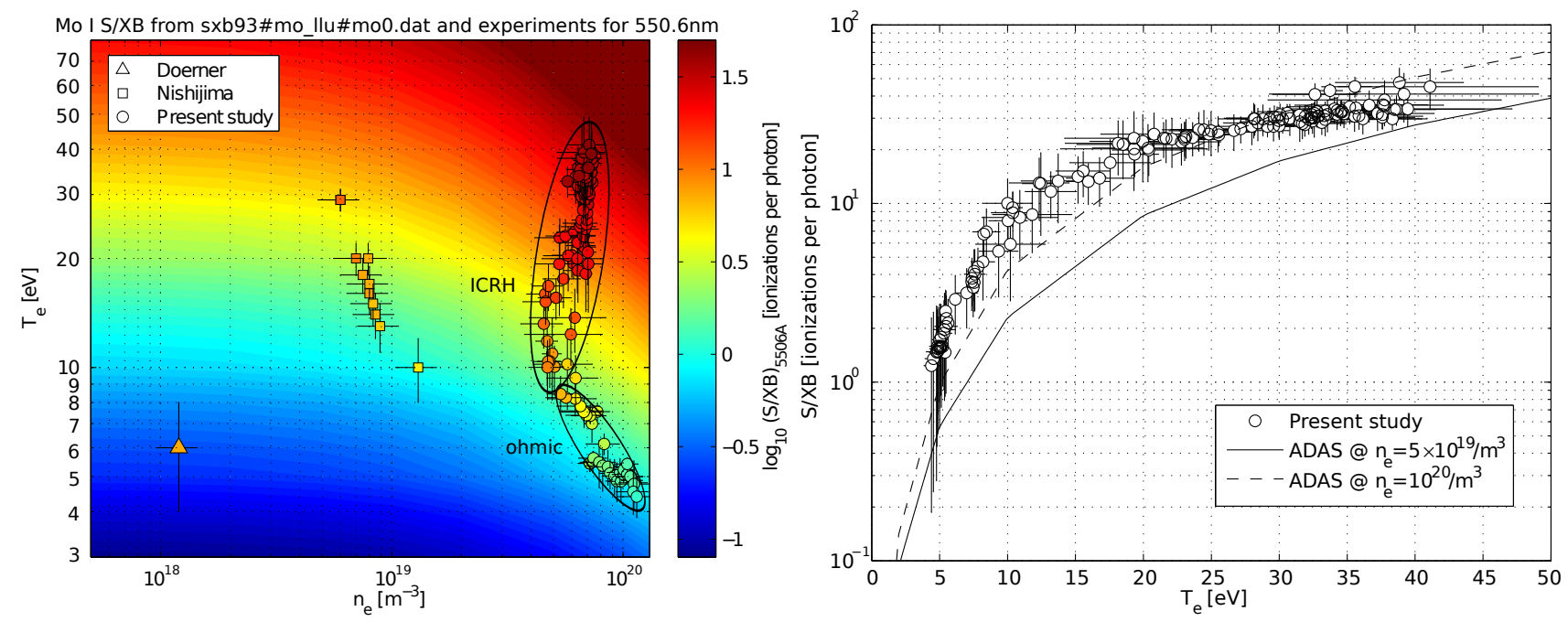

FIG. 10. Left: S/XB coefficients for the 550.6nm neutral molybdenum emission from 1993 ADAS low level unresolved (llu) calculations (colored contours) and empirical studies ${ }^{15,60}$ (points) with annotations of C-Mod operating ranges. Error bars shown for C-Mod data represent a combination of I-V characteristic fit error, photon statistics, and measured differences between the two shots. Right: Empirical S/XB values for the present study are plotted again vs. temperature with error bars on the $\mathrm{S} / \mathrm{XB}$. Note that the experimental values come from a range of densities, shown on the left 


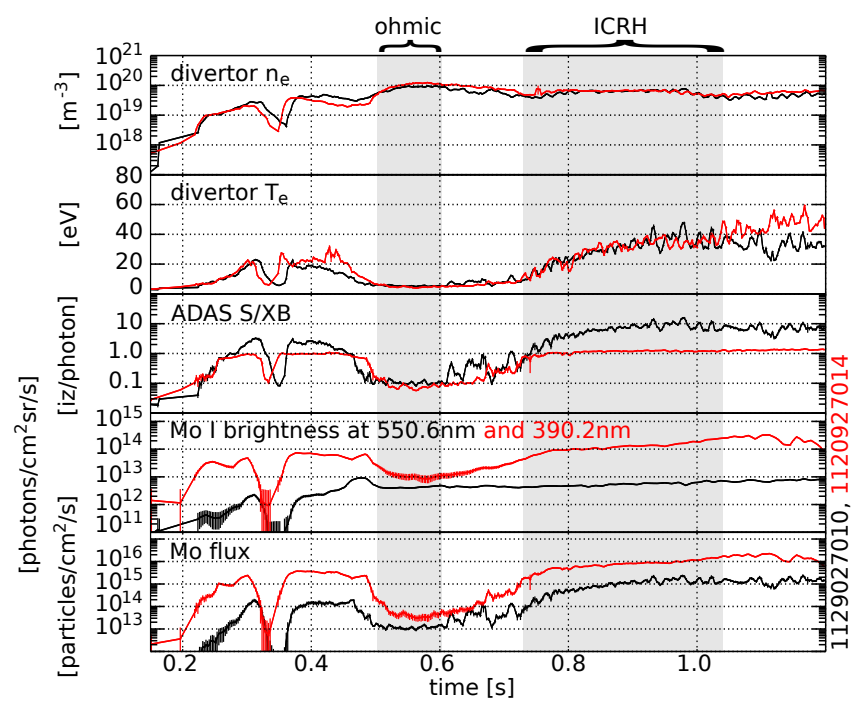

FIG. 11. Measurements near the outer divertor strike point of electron density and temperature from a Langmuir probe, S/XB value mapped from ADAS, atomic line brightness observed by Chromex, and associated molybdenum sputtering flux calculated from these during various phases of C-Mod operation. Data from the shaded regions is used to determine the semi-empirical $550.6 \mathrm{~nm}$ $\mathrm{S} / \mathrm{XB}$ values shown in figure 10 . 